\title{
Electrical Coupling of Heterotypic Ganglion Cells in the Mammalian Retina
}

\author{
(C) Christian Puller, ${ }^{\star}$ Sabrina Duda, ${ }^{\star}$ Elaheh Lotfi, ${ }^{\star}$ Yousef Arzhangnia, Christoph T. Block, Malte T. Ahlers, \\ and Martin Greschner \\ Department of Neuroscience and Research Centre Neurosensory Science, Carl von Ossietzky University, 26111 Oldenburg, Germany
}

Electrical coupling has been reported to occur only between homotypic retinal ganglion cells, in line with the concept of parallel processing in the early visual system. Here, however, we show reciprocal correlated firing between heterotypic ganglion cells in multielectrode array recordings during light stimulation in retinas of adult guinea pigs of either sex. Heterotypic coupling was further confirmed via tracer spread after intracellular injections of single cells with neurobiotin. Both electrically coupled cell types were sustained ON center ganglion cells but showed distinct light response properties and receptive field sizes. We identified one of the involved cell types as sustained $\mathrm{ON} \alpha$-ganglion cells. The presence of electrical coupling between heterotypic ganglion cells introduces a network motif in which the signals of distinct ganglion cell types are partially mixed at the output stage of the retina.

Key words: ganglion cell; gap junction; multielectrode array; reciprocal signaling; retina; tracer coupling

Significance Statement

The visual information is split into parallel pathways, before it is sent to the brain via the output neurons of the retina, the ganglion cells. Ganglion cells can form electrical synapses between dendrites of neighboring cells in support of lateral information exchange. To date, ganglion-to-ganglion cell coupling is thought to occur only between cells of the same type. Here, however, we show that electrical coupling between different types of ganglion cells exists in the mammalian retina. We provide functional and anatomical evidence that two different types of ganglion cells share information via electrical coupling. This new network motif extends the impact of the heavily studied coding benefits of homotypic coupling to heterotypic coupling across parallel neuronal pathways.

\section{Introduction}

The separation of visual information into parallel streams starts at the first retinal synapse formed between photoreceptors and more than a dozen distinct bipolar cell types (Euler et al., 2014). By the time the information leaves the retina via $>30$ types of retinal ganglion cells (RGCs; Baden et al., 2016), the visual information is separated into parallel pathways, in which each RGC type carries a distinct signal to the higher visual centers of the brain (Nassi and Callaway, 2009). Reciprocal electrical coupling between neurons via gap junctions is a recurrent scheme throughout the CNS including the retina (O'Brien and Bloom-

Received June 13, 2019; revised Nov. 18, 2019; accepted Dec. 18, 2019.

Author contributions: C.P. and M.G. designed research; C.P. and S.D. performed research; M.T.A. contributed unpublished reagents/analytic tools; C.P., S.D., E.L., Y.A., C.T.B., and M.G. analyzed data; C.P. and M.G. wrote the paper.

This work was supported by the VolkswagenStiftung. We thank Bettina Kewitz for technical assistance. We also thank Karin Dedek and Michael B. Manookin for comments on an earlier version of the manuscript.

*C.P., S.D., and E.L. contributed equally to this work.

The authors declare no competing financial interests.

Correspondence should be addressed to Christian Puller at christian.puller@uni-oldenburg.de.

https://doi.org/10.1523/JNEUROSCl.1374-19.2019

Copyright $\odot 2020$ the authors field, 2018). At the level of RGCs, examples of electrical coupling between cells of the same type (homotypic) are well known (Vaney, 1991; DeVries, 1999; Hu and Bloomfield, 2003; Hidaka et al., 2004; Trong and Rieke, 2008; Greschner et al., 2011; Trenholm et al., 2013, 2014; Völgyi et al., 2013), whereas functional heterotypic coupling of cells has so far only been reported to occur between RGCs and inhibitory interneurons (Ackert et al., 2006; Völgyi et al., 2013; Greschner et al., 2016). The same conclusions were drawn from tracer coupling patterns of neurobiotin-injected RGCs indicative of gap junction coupling in various species (Vaney, 1991; Dacey and Brace, 1992; Xin and Bloomfield, 1997; Schubert et al., 2005b; Völgyi et al., 2005; Zhang et al., 2005) as well as from electron microscopic reconstructions of synaptic circuits (Marc et al., 2018).

Here, we performed a large-scale analysis of spike correlation patterns between various cell types by multielectrode array (MEA) recordings of adult guinea pig retinas. We provide functional and anatomical evidence for heterotypic electrical coupling between two types of sustained ON center (sON) RGCs, which is not in line with the common notion of a functional separation of the neuronal pathways at the level of the retinal output. 


\section{Materials and Methods}

Animals and tissue preparation. All experiments were performed in accordance with the institutional guidelines for animal welfare and the laws on animal experimentation issued by the European Union and the German government. Guinea pigs of either sex were killed by an overdose of pentobarbital (Narcoren, Boehringer Ingelheim). Animals at an age of 3-35 months were used for the experiments, and no difference in the prevalence of homotypic or heterotypic RGC coupling was apparent. Animals were housed in a $12 \mathrm{~h}$ light/dark cycle, and experiments were performed during daytime hours.

Retinas were dissected from the eye-cup under infrared illumination in Ames' solution, pH 7.4 (Sigma-Aldrich) bubbled with carbogen (95\% $\mathrm{O}_{2}$ and $5 \% \mathrm{CO}_{2}$ ) at room temperature (RT). MEA recordings were performed at $34^{\circ} \mathrm{C}$ in the recording chamber. For neurobiotin injections, the tissue was stored under photopic light conditions in Ames' solution at $\sim 30^{\circ} \mathrm{C}$.

$M E A$ recordings and analysis. Retinas were recorded as described previously (Field et al., 2007). Briefly, a $3 \times 3 \mathrm{~mm}$ piece of isolated retina from the ventral half of the eye was mounted, ganglion cell side down, on a large-scale CMOS (complementary metal-oxide-semiconductor) array (3Brain). Recordings were analyzed offline to isolate the spikes of different cells. Candidate spike events were detected using a threshold on each electrode. The voltage waveforms on the electrode and neighboring electrodes around the time of the spike were extracted. Clusters of similar spike waveforms were identified as candidate neurons if they exhibited a refractory period. Duplicate recordings of the same cell were identified by temporal cross-correlation and removed.

A random noise stimulus with a natural spatiotemporal frequency falloff was used to characterize the response properties of recorded cells. The stimulus was presented on a CRT monitor at a refresh rate of $120 \mathrm{~Hz}$ and a stimulus pixel width of $49 \mu \mathrm{m}$ on the retina at photopic light levels at a mean intensity of $2.9 \mathrm{~mW} / \mathrm{m}^{2}$. Only the green and blue monitor guns were used. The receptive field was approximated by the spike-triggered average. Since the random noise stimulus was spatiotemporally correlated, the spike-triggered average does not represent an unbiased linear filter but produces a slightly blurred spatial filter. RGCs were functionally classified into types based on their spatiotemporal receptive field properties and spike autocorrelation function. Receptive field outlines were drawn at the 1 SD contour of two-dimensional Gaussian fits. Receptive field size estimates are reported as the diameter of a circle with the same area as the elliptical Gaussian fit. The receptive field areas of coupled and uncoupled RGCs and of the receptive fields calculated from synchronous and remaining spikes were estimated by the number of stimulus pixels above a threshold (one-third) of the peak amplitude. The distance between these receptive fields were estimated as the distance between the centroids of the selected stimulus pixel. This method had sufficient flexibility to measure asymmetric receptive fields compared with the symmetric Gaussian fit.

Cross-correlation functions were obtained by binning spikes and computing the correlation coefficient between the resulting spike count vectors, with a temporal offset. Cross-correlation functions were summarized by averaging across neighboring pairs showing reciprocal coupling. Cells with noisy spike-triggered averages, auto-correlation or cross-correlation functions, or electrical images indicative of imperfect spike sorting were excluded from the analysis. Cross-correlation functions were calculated over spike recordings of 50-60 min duration in the presence of a random noise stimulus. No apparent timing difference was observed between the left and right peaks in the cross-correlation functions of heterotypic pairs. Therefore, both peaks were included in the reported average offset times for homotypic and heterotypic pairs. For the conditional cross-correlation analysis (see Fig. 6), we calculated the linear response to the stimulus using the spike-triggered average as the linear filter. As mentioned above, the spike-triggered average of a spatiotemporally correlated stimulus is not an unbiased linear filter but produces a slightly blurred spatiotemporal version. We used this method for its familiarity in the field as no critical influence was expected for the resolution of this analysis. This was confirmed by a logistic regression model that is less sensitive to the stimulus correlations that led to quali- tatively similar results (data not shown). Quintiles of the linear responses were used to subdivide the spikes. We removed the slower correlations elicited by shared network noise, correlations of the light stimulus and artifacts introduced by the spike subdivision by subtracting the average cross-correlation function of uncoupled pairs with a similar average receptive field overlap and spike count.

The electrical image is the average spatiotemporal spike waveform recorded across the electrode array during the spikes detected from a specific cell (Litke et al., 2004; Field et al., 2007). The electrical image of a given cell was computed from a $10.1 \mathrm{~ms}$ window starting $4.48 \mathrm{~ms}$ before the peak negative voltage sample for each spike and was averaged over all recorded spikes. The spatiotemporal electrical image was further collapsed across time by taking the minimal voltage deflection at each electrode location, yielding the spatial representation seen in Figure 5, $A$ and $D$. For the analyses of the hot spots of correlated activity in the surrounding area of the soma of the cell, the electrical image was averaged from 2.1 to $0.39 \mathrm{~ms}$ before a spike. The analysis was robust over a wide range of time windows. The parameters were adjusted by hand to produce the strongest visually perceived contrast and to avoid any interference of the hot spot signals with those from central somatic spikes or overlapping dendrites. As the signals from the central somatic spikes were much stronger than those of the coupled cells in the hot spots, the time window was chosen with a sufficient distance to this peak. In addition, we chose a time window before the somatic spike, because the electrical activity spreading through the dendritic field overlapped in space and time with the hot spot activity, which hampered visualization of the hot spots in the two-dimensional image.

For the electrical image model, we used the full electrical image from the neighboring RGCs as a template for their electrical signature. If the neighboring RGCs fired a spike within a narrow time frame before or after a spike of the center cell, their electrical image was temporally shifted appropriately and added to the model electrical image. The mean of the model electrical image from 2.1 to $0.39 \mathrm{~ms}$ before a spike reproduced the hot spots of activity surrounding the soma of the cell. Next, the bimodal cross-correlation function between each neighbor and the center cell was fitted by the sum of three Gaussians. The bimodal peaks originate from reciprocal electrical coupling via gap junctions, and they are superimposed on slower correlations elicited by shared network noise and correlations of the light stimulus. We randomly shifted the spikes that contribute to the peak in the cross-correlation function, which occurs at approximately $-2 \mathrm{~ms}$ (seeFig. $5 \mathrm{H}$, inset) to remove correlated spikes that the neighbor cell fired before the center cell. In this partially decorrelated model, the amplitude of the hot spots was strongly reduced. This is consistent with the idea that the hot spots are mainly based on precise, gap junction-driven correlated spikes, while the waveforms of less precisely correlated spikes average out.

Neurobiotin injections. We performed intracellular injections of the gap junction-permeable tracer neurobiotin (Vaney, 1991). Pieces of isolated, ventral retinas were mounted on black nitrocellulose filter membranes with the ganglion cell layer up. For visualization of ganglion cell bodies, the tissue was incubated in acridine orange $(0.0001 \%$ in Ames' medium; Sigma-Aldrich) for $1 \mathrm{~min}$ and then mounted in the bath chamber where the tissue was continuously superfused with Ames' solution. Putative sON $\alpha$-ganglion cells were identified based on their large polygonal cell bodies in the ganglion cell layer under a $40 \times$ water-immersion objective (Zeiss). Intracellular injections were performed with sharp borosilicate glass electrodes (175-440 M $\Omega$ ). Electrodes were filled with a solution containing 10\% neurobiotin (Vector Laboratories) dissolved in $0.1 \mathrm{~m}$ Tris buffer, $\mathrm{pH} 7.3$, and $5 \mathrm{~mm}$ Invitrogen Alexa Fluor 568 hydrazide (Thermo Fisher Scientific). Electrodes were backfilled with $0.2 \mathrm{M} \mathrm{KCl}$. After the cell body was impaled and visualized by the Alexa Fluor dye fluorescence, neurobiotin was delivered by iontophoresis into the cell with a current of $0.6 \mathrm{nA}$ for $10 \mathrm{~min}$. After cell injections, the tissue was fixed for $20 \mathrm{~min}$ in $4 \%$ paraformaldehyde (PFA) in $0.01 \mathrm{M} \mathrm{PBS,} \mathrm{pH} \mathrm{7.4.}$ Following cryoprotection with $30 \%$ sucrose in PBS, retinas were stored at $-20^{\circ} \mathrm{C}$ until immunohistochemical labeling was performed.

Immunohistochemistry and light microscopy. For immunohistochemistry, the tissue was fixed in 4\% PFA in PBS for $20 \mathrm{~min}$ at RT. The tissue was cryoprotected overnight with $30 \%$ sucrose in PBS and stored at $-20^{\circ} \mathrm{C}$ 
sON alpha - sON alpha
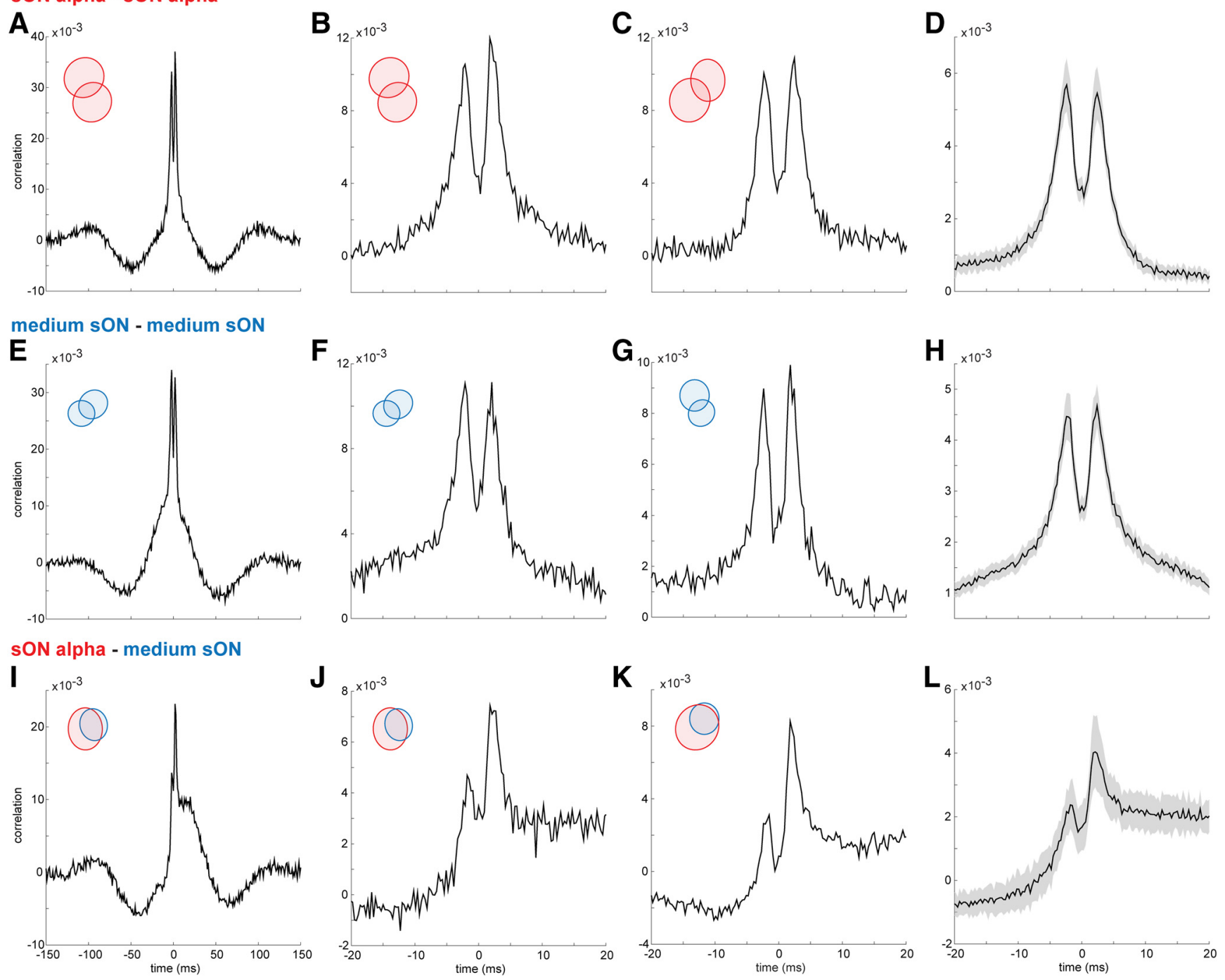

Figure 1. Cross-correlation functions of spiking activity between homotypic and heterotypic RGC pairs in one preparation. The retina was stimulated with a spatiotemporal random noise stimulus. $\boldsymbol{A}$, Cross-correlation function between two $\mathrm{s} 0 \mathrm{~N} \alpha$-RGCs. Insets show receptive field fits of the cell pair, as in Figure $2 A$. Bin size, $1 \mathrm{~ms}$. $\boldsymbol{B}$, Same cell pair as in $\boldsymbol{A}$ at a smaller time scale. Bin size, $0.3 \mathrm{~ms}$. C, Same as in $\boldsymbol{B}$ for a different cell pair. $\boldsymbol{D}$, Average cross-correlation function of neighboring sON $\alpha$-RGC pairs $(n=54)$. Gray shaded region represents the $95 \%$ confidence interval. $\boldsymbol{E}-\boldsymbol{H}$, As in $A-D$ for homotypic medium s0N pairs $(n=68)$. I-L, As in $A-D$ for heterotypic cell pairs of $50 \mathrm{~N} \alpha$ - and medium sON cells $(n=14)$. Time 0 indicates a spike of the s0N $\alpha$-RGC.

until use. Following dissection, retinal pieces were either used as whole mounts or sectioned vertically at $18-20 \mu \mathrm{m}$ using a cryostat (Leica).

Immunohistochemical labeling was performed by an indirect fluorescence method. Vertical sections were incubated overnight at RT with primary antibodies diluted in 5\% normal donkey serum (NDS), $0.5 \%$ Triton X-100, and $0.02 \%$ sodium azide in PBS. Sections were incubated for $1 \mathrm{~h}$ with secondary donkey antibodies diluted in the same incubation solution.

Retinal whole mounts were incubated at RT for 2-3 d in the primary antibody solution containing 5\% NDS, $1 \%$ Triton X-100 and $0.02 \%$ sodium azide dissolved in PBS. Primary antibodies used in this study were anti-RBPMS (rabbit, polyclonal, 1:500; catalog \#1830-RBPMS, PhosphoSolutions), anti-Neurofilament $\mathrm{H}$, nonphosphorylated (SMI32, mouse, monoclonal, 1:1000; catalog \#801701, BioLegend), anticholine acetyltransferase (ChAT; goat, polyclonal, 1:200; catalog \#AB144P, Merck). Secondary donkey antibodies (1:500, Invitrogen Alexa Fluor 488 and 568, Thermo Fisher Scientific; 1:250, Alexa Fluor 405, Abcam; 1:250, Cy5, Jackson ImmunoResearch; and 1:200, Invitrogen Alexa Fluor 568-conjugated streptavidin, Thermo Fisher Scientific) were incubated at RT for $4 \mathrm{~h}$ in the same incubation solution. The tissue was mounted on glass slides and coverslipped with VECTASHIELD (Vector
Laboratories). Spacers between glass slides and coverslips were used to avoid squeezing the tissue.

Image stacks were obtained with confocal laser-scanning microscopes (models TC SP8 and TCS SL, Leica). Overview scans were acquired with $10 \times$ or $20 \times$ air-objectives. High-resolution image stacks were acquired with $40 \times$ oil-immersion objectives (numerical aperture, $\geq 1.25$ ) and $z$-axis increments of $0.25-0.5 \mu \mathrm{m}$. Image stacks of neurobiotin-injected cells were median filtered in Fiji (Schindelin et al., 2012) to reduce photomultiplier noise (Kerschensteiner et al., 2009). Maximum intensity $x / y$ - or $z$-projections are shown in all figure panels of microscopic images and were performed in Fiji. The brightness and contrast of the final images were adjusted in Adobe Photoshop.

Cell bodies labeled with RBPMS or neurobiotin were manually outlined, and the corresponding areas were measured in Fiji to calculate the diameters. To analyze the location of tracer-coupled cells relative to the dendritic field of the injected cells, convex hulls were manually placed around the distal tips of individual dendrites in Fiji and the distances of the injected cell bodies to cell bodies of tracer-coupled cells relative to the hulls were measured.

Alignment and matching. After the MEA recordings were completed, images of the retina preparation on the multielectrode array were ob- 
tained with a Leica DM LFS epifluorescence microscope equipped with a $10 \times$ air-objective and manually aligned in Adobe Photoshop. Subsequently, the tissue was carefully mounted on black nitrocellulose filter membrane with the ganglion cell layer up. Following fixation, cryoprotection, and immunostaining, images of the labeled retina were obtained with a Leica DM6 B epifluorescence microscope equipped with a motorized stage and a $20 \times$ air-objective and automatically stitched together in the microscope software (LAS X, Leica). The outline of the tissue while mounted on the multielectrode array was aligned to the image of the successive immunostaining. sON $\alpha$-RGCs were identified in microscopic image stacks of these retinal whole mounts based on soma size, SMI-32 staining intensity, and stratification levels of the dendrites relative to the ChAT bands. The cells were then marked with the cell counter tool in Fiji in the center of their cell bodies.

To identify the corresponding pairs between recording locations of the large sON RGCs and the marked soma locations, a greedy algorithm was implemented. The recording location was defined as the weighted average of the electrode locations with significant somatic signals. For all recording locations, the distance to the second nearest soma was divided by the distance to the nearest soma. The pair with the largest distance ratio was selected as a corresponding pair. Next, all ratios were updated and the procedure was repeated. To minimize artifacts caused by the border of the preparation and by missing, unidentified cells, potential pairs with distances between the recording and soma location larger than the average nearest neighbor distance were excluded. The robustness of the greedy procedure was tested by the alignment optimization (see below) of random permutations of the nonflipped mosaics.

Next, we tested whether the observed alignment of recording and soma locations could have occurred by chance. To calculate the chance expectation, the mosaic of soma locations was flipped and transformed through random rotations and translations. The soma locations were rotated from $0^{\circ}$ to $360^{\circ}$ in 10 steps and shifted in $\mathrm{X}$ and $\mathrm{Y}$ direction up to two times the average nearest neighbor distance with steps of $10 \mu \mathrm{m}$. The mean distance between corresponding pairs in the observed alignment was smaller than in all random permutations of the flipped mosaic in two independent preparations (see Fig. 3I, black arrowhead). Therefore, the permutation analysis indicated that the match was inconsistent with chance alignment.

Finally, after testing the match of the two mosaics, the alignment was optimized to account for possible small deviations due to tissue handling and fixation. The scaling, position, and angle were adjusted to minimize the mean distance between recording and soma locations. For the preparation seen in Figure $3 \mathrm{H}$, the soma mosaic was scaled to $98 \%$, shifted 1.5 electrode positions in $\mathrm{X}$ and 0.4 in $\mathrm{Y}$ direction, and was not rotated from the original alignment to reduce the mean distance to $30 \mu \mathrm{m}$ (Fig. $3 I$, black arrow).

Statistical analyses. Statistical analyses comprised nonparametric tests. A Wilcoxon rank sum test or a Wilcoxon signed-rank test for paired comparisons was used to test for statistical significance. To test the observed alignment of recording positions and soma locations (Fig. 3I), a permutation analysis was performed as described above. Outliers were always included in the statistical analysis. Measurements are reported as the mean \pm SD. Shaded regions in figures represent the $95 \%$ confidence interval.

\section{Results}

Reciprocal firing of ganglion cells indicates homotypic and heterotypic coupling

We recorded the spiking activity of RGCs in adult guinea pig retinas with a large-scale multielectrode array to investigate their pattern of correlated electrical signaling. Reciprocal electrical coupling of RGCs via gap junctions causes highly correlated spiking activity that is reflected in a bimodal cross-correlation function with sharp peaks offset from 0 (Mastronarde, 1983; Brivanlou et al., 1998; DeVries, 1999; Völgyi et al., 2013). Homotypic electrical coupling was readily observed between neighboring cells of a large-size (Fig. $1 A-D$ ) and a medium-size (Fig. $1 E-H)$ type of sON RGCs among other types. Bimodal peaks
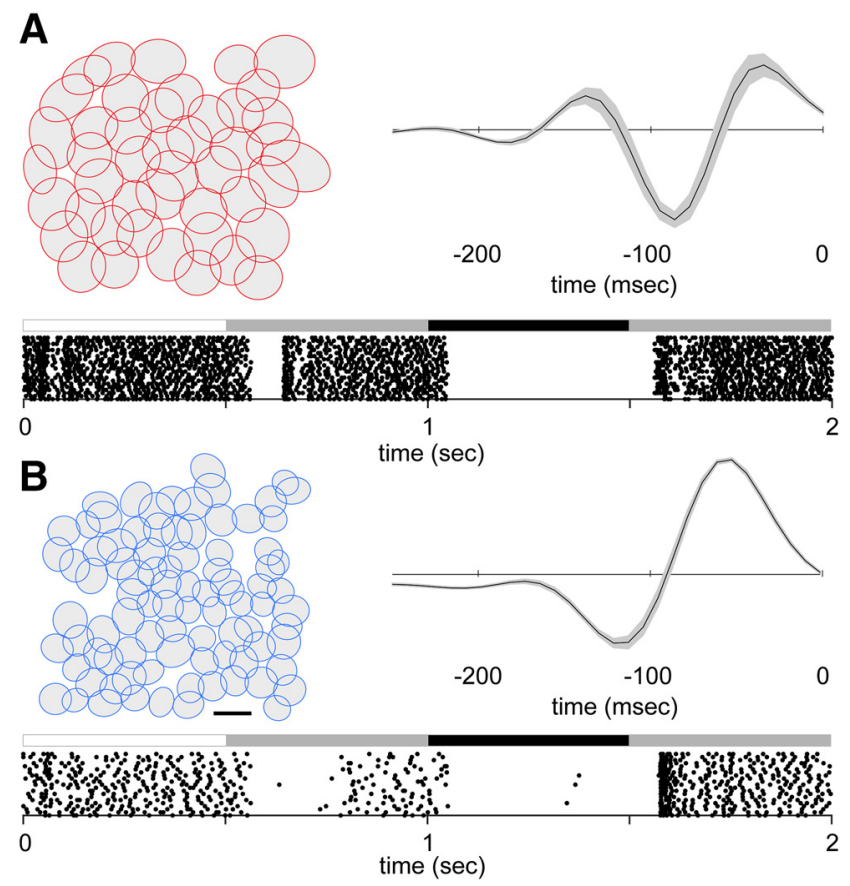

Figure 2. Receptive field mosaics of heterotypically coupled cell types. $\boldsymbol{A}$, Receptive field outlines of an sON $\alpha$-RGC mosaic (see Materials and Methods). Right, Mean spike-triggered average stimulus time course. Shaded region represents the $95 \%$ confidence interval. Bottom, Spike responses of an example $\mathrm{SON} \alpha$-cell to 20 repeats of full-field light intensity steps (indicated above the spike trains). $\boldsymbol{B}$, As in $\boldsymbol{A}$ for medium sON RGCs. Scale bars: $\boldsymbol{B}$ (for $\boldsymbol{A}, \boldsymbol{B}), 400 \mu \mathrm{m}$.

occurred with an offset of approximately $\pm 2 \mathrm{~ms}$ from 0 [medium sON, $2.4 \pm 0.4 \mathrm{~ms}, n=136$; large $\mathrm{sON}$ ( $\mathrm{sON} \alpha$, see below), $2.6 \pm$ $0.4 \mathrm{~ms}, n=108$; mean $\pm \mathrm{SD}$ ]. These pronounced peaks were superimposed on slower correlations elicited by shared network noise and correlations of the light stimulus during recordings (Trong and Rieke, 2008; Greschner et al., 2011). Intriguingly, the same pattern of correlation was observed for heterotypic pairs of the medium and large sON RGCs (Fig. $1 I-L$ ). Slow light-driven correlations were off centered, which pointed to a difference in the light response kinetics of the two cell types (Fig. 2). The bimodal peaks, however, were centered around 0 with a short delay of $2.1 \pm 0.6 \mathrm{~ms}(n=38)$, which indicated direct, functional coupling between two distinct RGC types. This heterotypic coupling pattern was cell type specific across preparations.

\section{Heterotypically coupled ganglion cells form two independent mosaics}

RGCs of individual types tile the retina with their dendritic trees and thus form mosaics with their receptive fields (Wässle et al., 1981). Accordingly, the receptive fields of the two types of heterotopically coupled RGCs formed two independent mosaics and showed distinct ON center light response properties (Fig. 2). The first cell type exhibited large receptive fields $(\varnothing=499 \pm 38 \mu \mathrm{m}$, $n=45)$ and a triphasic temporal filter, while the second cell type had medium-sized receptive fields $(\varnothing=313 \pm 35 \mu \mathrm{m}, n=89)$ and a more biphasic temporal filter. Both types showed sustained light responses to full-field light intensity steps.

\section{Identification of sON $\alpha$-ganglion cells as one of the heterotypically coupled types}

Next, we immunolabeled retinas after having recorded their physiological responses with the multielectrode array to reveal the morphological identity of one of the functional types (Li et al., 

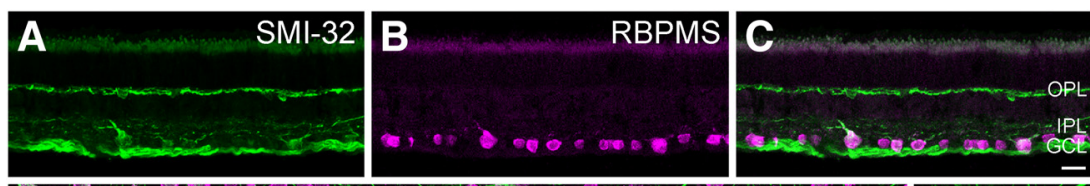

\section{H}
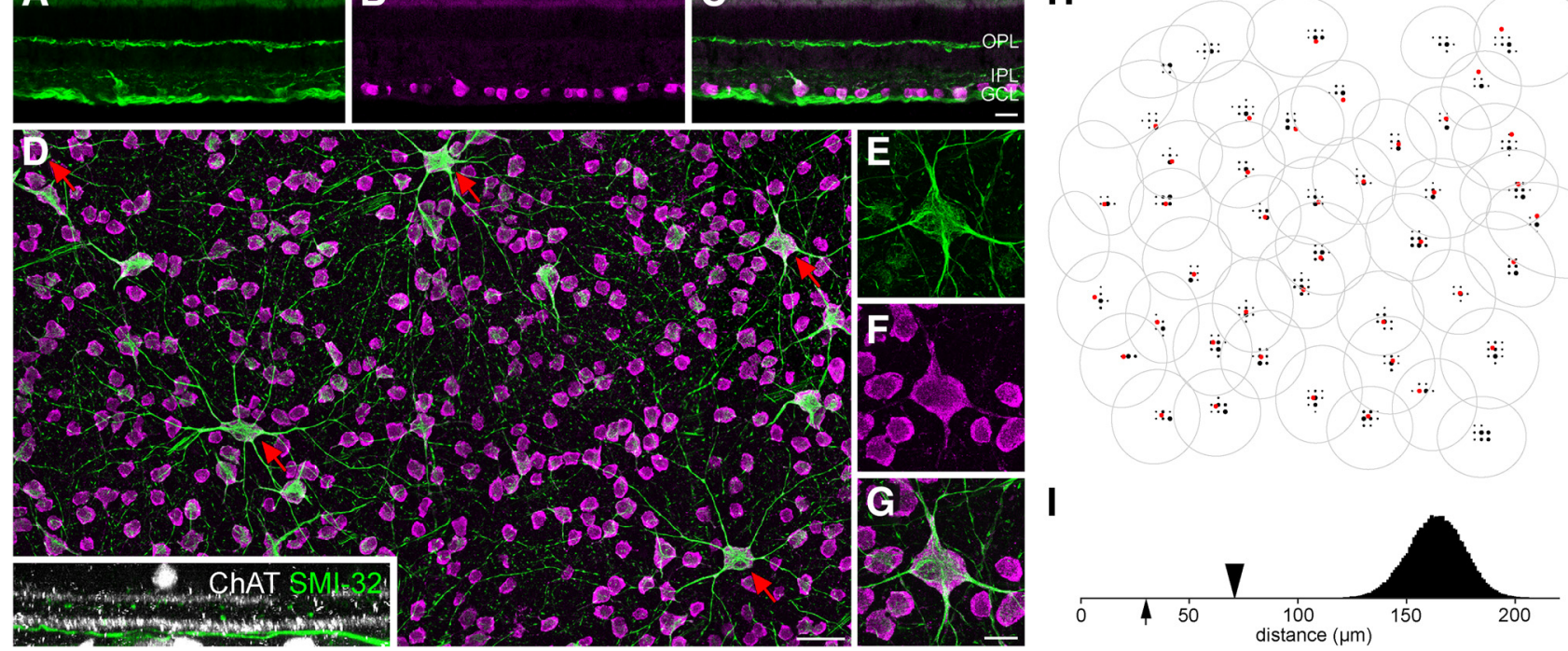

Figure 3. $S O \mathrm{~N} \alpha-\mathrm{RGCS}$ are heterotypically coupled. $\boldsymbol{A}-\boldsymbol{C}$, Vertical cryosection labeled with RBPMS and SMI-32. OPL, Outer plexiform layer; IPL, inner plexiform layer; GCL, ganglion cell layer. $\boldsymbol{D}$, Staining as in $\boldsymbol{A}-\mathrm{C}$ together with ChAT (inset) in a whole-mounted retina. Red arrows indicate $\mathrm{SON} \alpha$-RGCs. Inset shows the stratification of dendrites of an sON $\alpha$-RGC relative to the dendritic tier of starburst amacrine cells in an $x / y$-projection of $\boldsymbol{D}$. $\boldsymbol{E}$ - $\boldsymbol{G}$, Magnification of the $\mathrm{S} O \mathrm{~N} \alpha$-cell body in the top right corner of $\boldsymbol{D}$. $\boldsymbol{H}, 0$ verlay of soma locations from immunolabeled sON $\alpha$-cells (red dots), with strongest electrodes from recorded large $\mathrm{SON}$ type RGCS (black dots) and respective receptive field outlines (gray ellipses). I, Permutation analysis indicates a probable match of recorded cells with immunolabeling. Black arrowhead indicates the observed mean distance between cell somas and recording locations. Histogram represents the mean distances for $>165,000$ random permutations of the mosaic. Black arrow indicates the mean distance after optimization (see Materials and Methods). The distance between electrodes (black dots) is $42 \mu \mathrm{m}$. Scale bars: C, G, $20 \mu \mathrm{m}$; $D, 50 \mu \mathrm{m}$.

2015). Some of the functional features of the larger cell type resembled those of the $\mathrm{SON} \alpha$-RGCs of the guinea pig retina (Demb et al., 1999). sON $\alpha$-RGCs are intensely labeled with antibodies against the neurofilament marker SMI-32 in mice (Bleckert et al., 2014; Krieger et al., 2017). Therefore, we used SMI-32 in combination with RBPMS, which labeled all RGCs (Rodriguez et al., 2014), and ChAT to label starburst amacrine cells as reference points for dendritic stratification depth in the inner plexiform layer (Manookin et al., 2008). In addition to horizontal cells, several types of RGCs were labeled with SMI-32 similar to the mouse retina (Fig. $3 A-G$ ). Among the most intensely labeled cells, an ON RGC type had polygonal-shaped cell bodies, which were remarkably large ( $\varnothing=29 \pm 2 \mu \mathrm{m}, n=52)$ when compared with all other cell bodies $(\varnothing=17 \pm 3 \mu \mathrm{m}, n=714)$. In addition, the primary dendrites of these cells stratified just beneath the dendritic plexus of ON starburst amacrine cells. These features were highly suggestive of the sON $\alpha$-RGCs in the guinea pig retina (Demb et al., 1999; Manookin et al., 2008; Beaudoin et al., 2019). The putative sON $\alpha$-RGC cell body locations matched the recording locations of the large $\mathrm{SON}$ type derived from the electrical signals of the cells on the multielectrode array (Fig. $3 \mathrm{H}, \mathrm{I}$, permutation analyses: $p<0.001)$. Thus, the results suggested that one of the cell types of interest corresponds to the sON $\alpha$-RGCs.

Tracer injections of sON $\alpha$-ganglion cells reveal homotypic and heterotypic coupling

We performed intracellular injections of the gap junctionpermeable tracer neurobiotin (Vaney, 1991) to further confirm the heterotypic coupling of the sON $\alpha$-RGC to another RGC type. sON $\alpha$-RGCs were targeted with sharp microelectrodes based on their cell body shape and size. The characteristic morphology and dendritic stratification levels (Fig. 4A) confirmed that the targeted cells $(n=14)$ were sON $\alpha$-RGCs (Demb et al., 1999; Manookin et al., 2008; Beaudoin et al., 2019).
The tracer-coupling pattern revealed additional neurobiotinpositive cell bodies, which were all located in the RGC layer. Large, strongly SMI-32-labeled cell bodies $(\varnothing=26 \pm 2 \mu \mathrm{m}, n=$ 46 ; observed maximum coupled to one cell $=6$ ) were observed at the outer perimeter of the dendritic tree of the injected $\mathrm{sON}$ $\alpha$-cells (Fig. $4 A$, red arrows, $B-E$ ) in agreement with the homotypic coupling patterns between $\alpha$-like RGCs in other species (Vaney, 1991; Hidaka et al., 2004; Völgyi et al., 2005). Mediumsized cell bodies $(\varnothing=17 \pm 2 \mu \mathrm{m}, n=12$, observed maximum coupled to one cell $=5$ ) that were positive for RBPMS but only weakly labeled with SMI-32 were consistently located closer toward the cell bodies of the injected sON $\alpha$-RGCs ( $49 \pm 17 \%$ along the radial axes; Fig. $4 A$, blue arrow, $F-I$ ). These significantly smaller cell bodies ( $p<0.001$, Wilcoxon rank sum test) belonged to heterotypically coupled RGCs, most likely the medium sON RGCs. Additionally, small-diameter cell bodies, which did not colocalize with RBPMS, were observed and therefore corresponded to electrically coupled, displaced amacrine cells (Fig. 4A, black arrowheads, $J-M$ ).

\section{Electrical images show homotypic and heterotypic coupling patterns}

Heterotypic electrical coupling between sON $\alpha$-RGCs and medium sON RGCs was also evident from the electrical images (i.e., the spike-triggered electrical activity across all electrodes; Fig. 5). The electrical image of individual RGCs showed the expected spatial organization ( $\mathrm{Li}$ et al., 2015), which is a high-amplitude somatic area, a lower-amplitude dendritic area, and an axon extending toward the optic disk (Fig. 5A,D). However, if electrical signals were averaged only across a short time window before the somatic spike occurred, pronounced additional areas of electrical signals with larger amplitudes (hot spots) became visible (Fig. $5 B, C, E, F)$. These hot spots coincided with the location of neighboring homotypic (medium sON) and heterotypic ( $\mathrm{sON} \alpha$ ) 

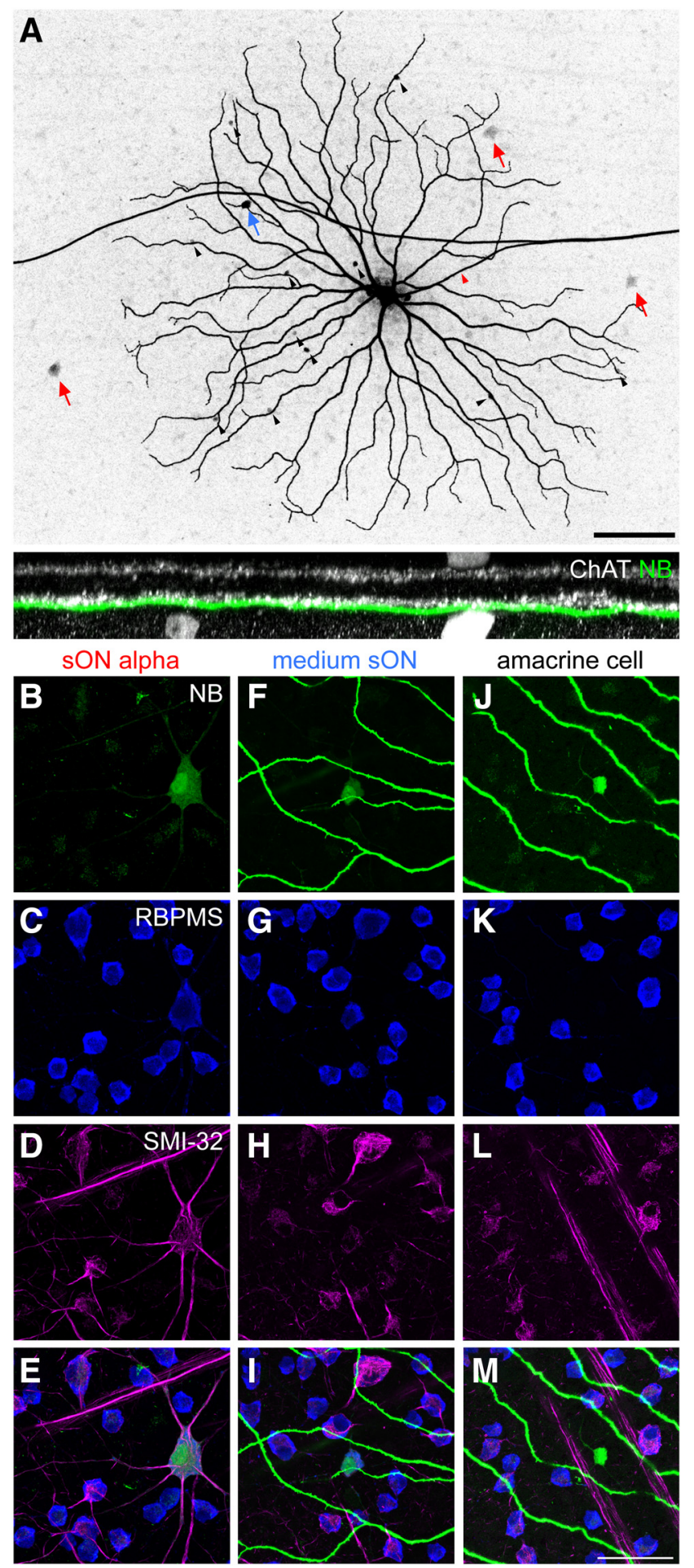

Figure 4. $\quad \mathrm{s} O \mathrm{~N} \alpha-\mathrm{RGCs}$ are tracer coupled to a heterotypic RGC type. $\boldsymbol{A}$, Top, An injected sON $\alpha$-RGC shows neurobiotin (NB) spread into large (red arrows), medium (blue arrow), and small (black arrowheads) cell bodies. The axon of the injected cell (red arrowhead) converges to an axon of another NB-injected, neighboring sON $\alpha$-RGC, which passes horizontally through the image. Bottom, The $x / y$-projection of a dendrite of the same cell relative to ChAT labeling. $\boldsymbol{B}-\boldsymbol{E}$, Magnified view of the leftmost NB-positive RGC body in $\boldsymbol{A}$ (red arrow), counterstained with RBPMS and SMI-32. $\boldsymbol{F}-\boldsymbol{I}$, As in $\boldsymbol{B}-\boldsymbol{E}$ for the cell body marked with a blue arrow in $\boldsymbol{A}$. $\boldsymbol{J}-\boldsymbol{M}$, As in $\boldsymbol{B}$ for an exemplary amacrine cell body. Scale bars: $\boldsymbol{A}, 150 \mu \mathrm{m} ; \boldsymbol{M}, 50 \mu \mathrm{m}$.

RGCs and thereby revealed the spatial organization of the coupling pattern. Medium sON RGCs were coupled to multiple neighboring sON $\alpha$-RGCs and medium sON RGCs (Fig. $5 C, F$ ). A simple model was used to test the idea that the hot spots are mainly based on the gap junction-driven correlations, while the signals of the less precisely correlated spikes average out. The hot spots could be reproduced using the electrical signatures of the neighboring cells from their electrical images and their spike correlations (Fig. 5G). As expected, the hot spot amplitude was strongly reduced when the precise gap junction-driven correlations were removed (Fig. $5 \mathrm{H}$, inset).

\section{Stimulus-dependent modulation of heterotypic reciprocal firing}

To get some insights into the interaction of heterotypic coupling and stimulus processing, we searched for stimulus conditions that increased or decreased the coupling. As above, we used a random noise stimulus with a natural spatiotemporal frequency falloff as a generic visual stimulus. First, we used the spatiotemporal receptive field of the medium sON RGCs to predict their linear response to the stimulus. Then, the linear prediction was used to subdivide cross-correlation functions according to the amount of stimulus-driven activation the cells received (Fig. 6). We removed the slower correlations elicited by shared network noise and correlations of the light stimulus by subtracting the average cross-correlation function of uncoupled pairs with similar receptive field overlap. Reciprocal firing increased when medium sON RGCs were increasingly activated by the visual stimulus. This was expected from previous observations of homotypic coupling properties (Mastronarde, 1983; Trong and Rieke, 2008; Trenholm et al., 2014). Dividing the cross-correlation functions based on the stimulus-driven activation of the sON $\alpha$-RGCs led to qualitatively identical results (data not shown). Instances in which one cell of the coupled pair was strongly activated by the stimulus while the other cell was inhibited hardly occurred due to the high receptive field overlap of the coupled pairs and their similar response properties.

sON $\alpha$-ganglion cells influence spiking in medium sON cells more effectively than vice versa

Across all stimulus conditions, the left peak of the crosscorrelation function is smaller than the right one (Fig. 6; i.e., a spike in sON $\alpha$-RGCs led to a spike in the medium sON RGCs more effectively than vice versa). Therefore, the question arose whether the medium sON RGC thereby inherited some of the stimulus filter properties of the coupled $\mathrm{sON} \alpha$-RGC. Indeed, the receptive fields of heterotopically coupled medium sON RGCs were larger than those of neighboring medium sON RGCs that showed no detectable heterotypic coupling $(134 \pm 17 \%$ area; $n=$ $12, p<0.001$, Wilcoxon rank sum test). To test whether this increase in receptive field size was consistent with being elicited by coupling, spatiotemporal receptive fields of medium sON RGCs were calculated only from spikes that occurred shortly after spikes in the sON $\alpha$-RGCs. The receptive fields were larger than those calculated from the remaining spikes $(127 \pm 31 \%$ area; $n=$ $14, p<0.002$, Wilcoxon signed-rank test). Furthermore, these medium $\mathrm{SON}$ receptive fields were extended toward the coupled sON $\alpha$-RGC, as indicated by a reduced distance between their receptive field centers $(76 \pm 18 \% ; n=14, p<0.001$, Wilcoxon signed-rank test).

\section{Discussion}

In summary, the correlated spiking activity, tracer coupling, and spatial patterns of shared electrical activity in the electrical images all pointed to heterotypic coupling between two distinct types of RGCs. 

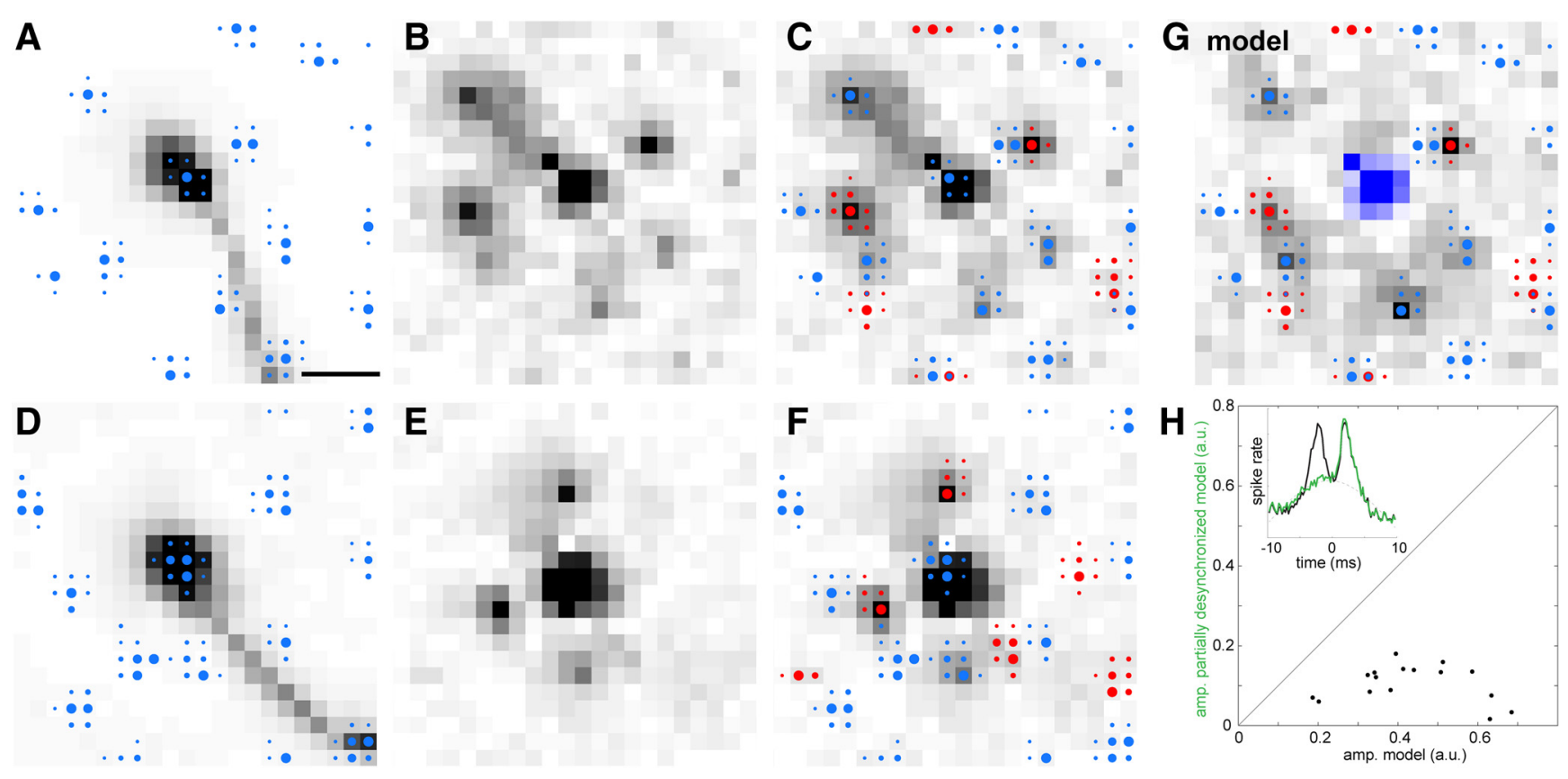

Figure 5. The electrical images of the medium SON RGCs revealed the homotypic and heterotypic coupling pattern. $A$, The minimum projection of the spike-triggered electrical activity of a medium sON RGC from 4.48 ms before to $6.72 \mathrm{~ms}$ after the somatic spike. Darker intensities indicate more negative voltage deflections. Largest amplitudes are saturated to increase visibility. Clusters of blue dots mark the electrode locations with the strongest signals for individual medium sON RGCs. $\boldsymbol{B}$, The mean spike-triggered electrical activity from 2.1 to $0.39 \mathrm{~ms}$ before a spike of the cell in $\boldsymbol{A}$ reveals hot spots of activity surrounding the soma of the cell. $\boldsymbol{C}$, As in $\boldsymbol{B}$ with the locations of strong signals of medium sON RGCs (blue dots, as in $\boldsymbol{A}$ ) and sON $\alpha$-RGCs (red dots). $\boldsymbol{D}-\boldsymbol{F}$, As in $\boldsymbol{A}-\boldsymbol{C}$ for a different medium sON RGC. G, Model of the medium sON RGC hot spots in $\boldsymbol{C}$, based on the highly synchronized spikes and the somatic mean waveforms of neighboring cells, reproduces the correlation pattern. The center RGC position was marked with blue squares according to its appearance in $\boldsymbol{B}$ and $\boldsymbol{C}$ for visual guidance. $\boldsymbol{H}$, Scatterplot of the hot spot amplitudes in the original model (G) versus a partially desynchronized model. Inset, Original cross-correlation function (black) of a SON $\alpha$ pair and their cross-correlation function after the partial removal of precise correlations (green). The dashed line is a Gaussian fit to the slower correlation component. Amplitude is given in arbitrary units. Scale bars: $\boldsymbol{A}$ (for $\boldsymbol{A}-\boldsymbol{G}), 200 \mu \mathrm{m}$.

\section{quintiled linear response to the stimulus}

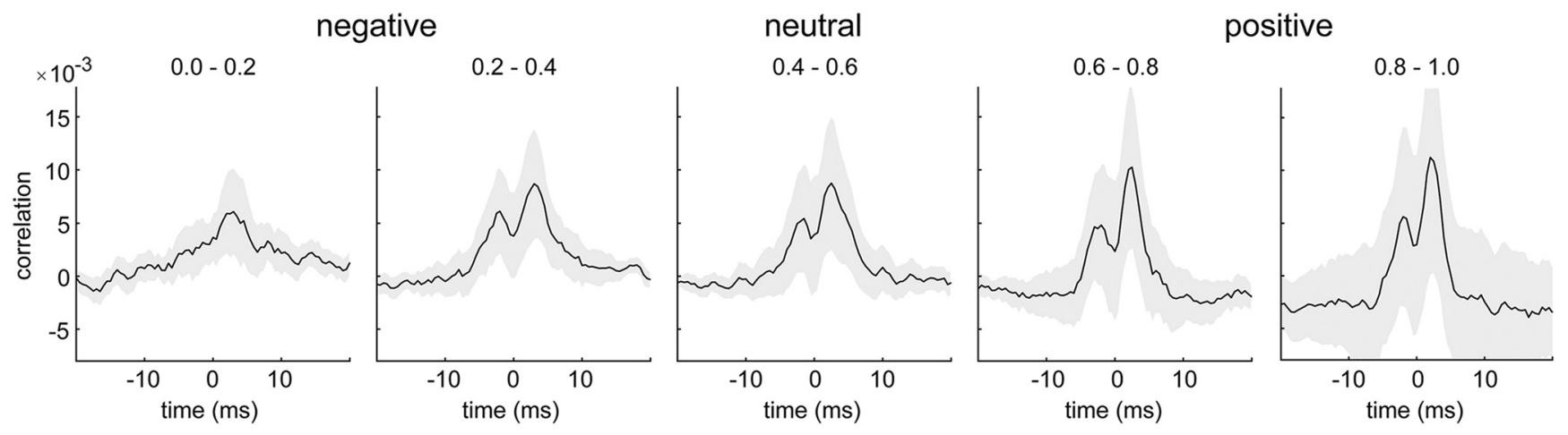

Figure 6. Heterotypic reciprocal firing of RGCs is modulated by their stimulus-driven activation. Cross-correlation function between cell pairs of sON $\alpha$-RGCs and medium sON RGCs conditional on the stimulus-driven activation of the medium SON RGCs. The stimulus-driven activation was estimated as the linear response to the stimulus. Quintiles of the linear response were used to subdivide the spikes. Stronger activation from left to right. Stimulus and noise correlations were removed by subtracting the average cross-correlation function of uncoupled pairs with similar receptive field overlap. Data are as in Figure $1 L(n=14)$. Time 0 indicates a spike of the $\mathrm{SON} \alpha$-RGC. Gray shaded region represents the $95 \%$ confidence interval. Cross-correlation function bin width, $2 \mathrm{~ms}$; bin center shift, $0.5 \mathrm{~ms}$.

\section{Electrical coupling via gap junctions}

The narrow, bimodal peaks in cross-correlation functions of spiking activity between heterotypic RGC pairs presented here closely resemble those of homotypic pairs in our study and previously published examples in terms of amplitude and timing (Mastronarde, 1983; Brivanlou et al., 1998; DeVries, 1999; Trong and Rieke, 2008; Völgyi et al., 2013). The fast reciprocal firing has been attributed to gap junction coupling of the corresponding cells based on temporal firing properties and pharmacological manipulations. The bimodal shape indeed reflects direct reciprocal RGC coupling via gap junctions formed by connexins (Völgyi et al., 2013). In addition, tracer coupling of RGCs is commonly interpreted as evidence of gap junction coupling via connexins (Vaney, 1991; Dacey and Brace, 1992; Xin and Bloomfield, 1997; Zhang et al., 2005). This was confirmed in studies of genetically modified mice lacking the connexin subunit proteins Cx36 or Cx45 (Schubert et al., 2005a,b; Pan et al., 2010; Völgyi et al., 2013; Roy et al., 2017). Cx36 is the major connexin subunit involved in RGC coupling (Pan et al., 2010) in addition to Cx45 and possibly Cx30.2 (Schubert et al., 2005a; Müller et al., 2010), and the guinea pig retina shows a distribution of $\mathrm{Cx} 36$ common to mammals (Kovács-Öller et al., 2017). Therefore, it is likely that heterotypic 
coupling between sON $\alpha$-RGCs and medium sON RGCs occurs via direct, connexin-containing gap junctions between the two cell types, while the connexin subunit that mediates the electrical coupling of these cell types remains unknown. We observed a bias toward medium sON cells in the spiking probability during reciprocal firing (Figs. 1, 6), which could possibly reflect rectification caused by differences in input resistance or membrane capacitance between the two cell types (Veruki and Hartveit, 2002), with the latter caused by different cell sizes (i.e., sON $\alpha$-cells being larger than medium sON cells as estimated from cell body and receptive field sizes; Figs. 2, 3).

\section{Coupling patterns of ganglion cells}

Even though homotypic coupling has been known for decades, heterotypic electrical coupling between RGCs has not been observed in previous studies. Similarly, electrical coupling of neurons in other parts of the CNS is thought to occur in a homotypic manner (Traub et al., 2018). Based on our investigations, homotypic electrical coupling is a common phenomenon in the guinea pig retina, which occurs among several types of ganglion cells, similar to what has been shown in other species (O'Brien and Bloomfield, 2018). The coupling pattern of sON $\alpha$-RGCs, however, is not consistent when compared across species. Homotypic coupling was not observed between ON $\alpha$-RGCs of mouse and rabbit retinas (Xin and Bloomfield, 1997; Völgyi et al., 2005; Zhang et al., 2005). In contrast, ON $\alpha$-RGCs were shown to be homotypically coupled in rat and ferret (Penn et al., 1994; Hidaka et al., 2004). While the analogies between rodent ON $\alpha$ - and primate ganglion cell types are still under debate, ON parasol cells are a well known example for homotypically coupled RGCs in primates (Dacey and Brace, 1992; Trong and Rieke, 2008; Greschner et al., 2014). Therefore, our findings regarding the heterotypic coupling of sON $\alpha$-RGCs in guinea pig retinas cannot be treated as a general rule for electrical coupling of this cell type, as coupling patterns greatly differ between species. Nevertheless, it is likely that heterotypic electrical coupling of RGC types exists across species. Functional analyses across many cells in conjunction with a reliable cell type classification, which have rarely been conducted in the past, are required to reveal these interactions between pathways. Evidence for heterotypic RGC coupling in previous tracer injections may have been missed because of the complex coupling patterns, with labeled cell bodies of different shapes and sizes. Tracer-coupled cell bodies that did not match the injected cell body size were by default identified as large amacrine cells, where additional molecular markers would have been required to reveal the identity of cells. Finally, the interpretation of results may have been further confounded by the prevailing premise of RGC types never being heterotypically coupled to preserve their function as parallel and independent processing units.

\section{Functional roles of electrical coupling}

Insight into the functional roles of reciprocal coupling at the level of RGCs was gained from studies of homotypic coupling among certain RGC types. The coding benefits have been shown to include enhanced signal-to-noise ratio and stimulus saliency (Bloomfield and Völgyi, 2009; Trenholm et al., 2013; Yao et al., 2018). The heterotypic coupling of RGCs described here introduces a new network motif that extends these mechanisms across parallel output channels. Moreover, heterotypic coupling may give rise to an efficient circuitry, where certain stimulus features are passed on from one pathway to another only after the filter properties of the spiking nonlinearity. Heterotypically coupled cells may take advantage of this feature-selective filter residing in their coupled partners, as it does not exist in the presynaptic circuitries of the cells. First, the stimulus features must match the preferred filter properties of the cells to overcome the strong nonlinearity of the spike generation. Then, if the spike threshold has been reached in one of the cells, a spike in the second, electrically coupled cell is produced only if its membrane potential is already close to threshold (Mastronarde, 1983; Trong and Rieke, 2008; Trenholm et al., 2014), which is further supported by the results of our study (Fig. 6). Due to stimulus correlations in natural scenes, it is common for overlapping or neighboring RGCs with similar response properties to receive excitatory inputs in close temporal relation, correlating their membrane potentials. This is the case for the sON $\alpha$-RGCs and medium sON RGCs as they show similar response properties and extensive stimulusdriven correlations (Figs. $1 I-L, 2$ ). In addition, the receptive field overlap between heterotypically coupled pairs is on average even higher than in homotypic pairs, which occurs due to the relative placement of the coupled neurons reflected in the mosaic structure of two independent ganglion cell types (Figs. 1 insets, 2, 4). Our results suggest that the medium sON RGCs inherit some of the receptive field features of sON $\alpha$-RGCs (Fig. 6). It is therefore tempting to speculate that information is passed from one cell type to another, which may not be available from their presynaptic circuitries. This could be the case for melanopsin signals, for instance, which are generated only at the level of certain ganglion cell types, including sON $\alpha$-RGCs in mice (Estevez et al., 2012; Schmidt et al., 2014).

\section{Interactions across neuronal pathways}

The optic nerve represents a bottleneck in the flow of visual information from the retina to the visual centers of the brain. Therefore, the output of the retina is bound to efficiently use the limited information capacity and is thought to minimize the redundancies of the signals. One consequence of this is the concept of parallel processing in the retina (Wässle, 2004; Nassi and Callaway, 2009). Initially, it is a confusing finding that, after the visual information was segregated with considerable effort into separate pathways, signals are partially mixed again at the output neurons even though there are many instances of potential cross talk within the retinal circuitry. However, the concept of parallel pathways in the retina is not meant in this absolute way, as demonstrated, for instance, by the cross talk in the inner retina even across ON and OFF pathways (Zaghloul et al., 2003; Hoshi et al., 2009) and the convergence of retinal pathways in the lateral geniculate nucleus (Rompani et al., 2017; Román Rosón et al., 2019). Therefore, a full assessment of the independence of channels is only possible after the complete circuitries, including the projection targets of the cells and the upstream processing of the signals, are understood. Overall, while we demonstrate here only a single example of heterotypic RGC-to-RGC coupling, our study serves as a counterexample to the common notion of exclusively homotypic coupling, and it shows that the complexity of electrical coupling across neuronal pathways has been underestimated in the previous literature.

\section{References}

Ackert JM, Wu SH, Lee JC, Abrams J, Hu EH, Perlman I, Bloomfield SA (2006) Light-induced changes in spike synchronization between coupled ON direction selective ganglion cells in the mammalian retina. J Neurosci 26:4206-4215.

Baden T, Berens P, Franke K, Román Rosón M, Bethge M, Euler T (2016) The functional diversity of retinal ganglion cells in the mouse. Nature 529:345-350.

Beaudoin DL, Kupershtok M, Demb JB (2019) Selective synaptic connections in the retinal pathway for night vision. J Comp Neurol 527:117-132. 
Bleckert A, Schwartz GW, Turner MH, Rieke F, Wong RO (2014) Visual space is represented by nonmatching topographies of distinct mouse retinal ganglion cell types. Curr Biol 24:310-315.

Bloomfield SA, Völgyi B (2009) The diverse functional roles and regulation of neuronal gap junctions in the retina. Nat Rev Neurosci 10:495-506.

Brivanlou IH, Warland DK, Meister M (1998) Mechanisms of concerted firing among retinal ganglion cells. Neuron 20:527-539.

Dacey DM, Brace S (1992) A coupled network for parasol but not midget ganglion cells in the primate retina. Vis Neurosci 9:279-290.

Demb JB, Haarsma L, Freed MA, Sterling P (1999) Functional circuitry of the retinal ganglion cell's nonlinear receptive field. J Neurosci 19:9756-9767.

DeVries SH (1999) Correlated firing in rabbit retinal ganglion cells. J Neurophysiol 81:908-920.

Estevez ME, Fogerson PM, Ilardi MC, Borghuis BG, Chan E, Weng S, Auferkorte ON, Demb JB, Berson DM (2012) Form and function of the M4 cell, an intrinsically photosensitive retinal ganglion cell type contributing to geniculocortical vision. J Neurosci 32:13608-13620.

Euler T, Haverkamp S, Schubert T, Baden T (2014) Retinal bipolar cells: elementary building blocks of vision. Nat Rev Neurosci 15:507-519.

Field GD, Sher A, Gauthier JL, Greschner M, Shlens J, Litke AM, Chichilnisky EJ (2007) Spatial properties and functional organization of small bistratified ganglion cells in primate retina. J Neurosci 27:13261-13272.

Greschner M, Shlens J, Bakolitsa C, Field GD, Gauthier JL, Jepson LH, Sher A, Litke AM, Chichilnisky EJ (2011) Correlated firing among major ganglion cell types in primate retina. J Physiol 589:75-86.

Greschner M, Field GD, Li PH, Schiff ML, Gauthier JL, Ahn D, Sher A, Litke AM, Chichilnisky EJ (2014) A polyaxonal amacrine cell population in the primate retina. J Neurosci 34:3597-3606.

Greschner M, Heitman AK, Field GD, Li PH, Ahn D, Sher A, Litke AM, Chichilnisky EJ (2016) Identification of a retinal circuit for recurrent suppression using indirect electrical imaging. Curr Biol 26:1935-1942.

Hidaka S, Akahori Y, Kurosawa Y (2004) Dendrodendritic electrical synapses between mammalian retinal ganglion cells. J Neurosci 24:10553-10567.

Hoshi H, Liu WL, Massey SC, Mills SL (2009) ON inputs to the OFF layer: bipolar cells that break the stratification rules of the retina. J Neurosci 29:8875-8883.

Hu EH, Bloomfield SA (2003) Gap junctional coupling underlies the shortlatency spike synchrony of retinal alpha ganglion cells. J Neurosci 23:6768-6777.

Kerschensteiner D, Morgan JL, Parker ED, Lewis RM, Wong RO (2009) Neurotransmission selectively regulates synapse formation in parallel circuits in vivo. Nature 460:1016-1020.

Kovács-Öller T, Debertin G, Balogh M, Ganczer A, Orbán J, Nyitrai M, Balogh L, Kántor O, Völgyi B (2017) Connexin36 expression in the mammalian retina: a multiple-species comparison. Front Cell Neurosci 11:65.

Krieger B, Qiao M, Rousso DL, Sanes JR, Meister M (2017) Four alpha ganglion cell types in mouse retina: function, structure, and molecular signatures. PLoS One 12:e0180091.

Li PH, Gauthier JL, Schiff M, Sher A, Ahn D, Field GD, Greschner M, Callaway EM, Litke AM, Chichilnisky EJ (2015) Anatomical identification of extracellularly recorded cells in large-scale multielectrode recordings. J Neurosci 35:4663-4675.

Litke AM, Bezayiff N, Chichilnisky EJ, Cunningham W, Dabrowski W, Grillo AA, Grivich MI, Grybos P, Hottowy P, Kachiguine S, Kalmar RS, Mathieson K, Petrusca D, Rahman M, Sher A (2004) What does the eye tell the brain? Development of a system for the large-scale recording of retinal output activity. IEEE Trans Nucl Sci 51:1434-1440.

Manookin MB, Beaudoin DL, Ernst ZR, Flagel LJ, Demb JB (2008) Disinhibition combines with excitation to extend the operating range of the OFF visual pathway in daylight. J Neurosci 28:4136-4150.

Marc RE, Sigulinsky CL, Pfeiffer RL, Emrich D, Anderson JR, Jones BW (2018) Heterocellular coupling between amacrine cells and ganglion cells. Front Neural Circuits 12:90.

Mastronarde DN (1983) Interactions between ganglion-cells in cat retina. J Neurophysiol 49:350-365.

Müller LP, Dedek K, Janssen-Bienhold U, Meyer A, Kreuzberg MM, Lorenz S, Willecke K, Weiler R (2010) Expression and modulation of connexin 30.2 , a novel gap junction protein in the mouse retina. Vis Neurosci 27:91-101.

Nassi JJ, Callaway EM (2009) Parallel processing strategies of the primate visual system. Nat Rev Neurosci 10:360-372.
O'Brien J, Bloomfield SA (2018) Plasticity of retinal gap junctions: roles in synaptic physiology and disease. Annu Rev Vis Sci 4:79-100.

Pan F, Paul DL, Bloomfield SA, Völgyi B (2010) Connexin36 is required for gap junctional coupling of most ganglion cell subtypes in the mouse retina. J Comp Neurol 518:911-927.

Penn AA, Wong RO, Shatz CJ (1994) Neuronal coupling in the developing mammalian retina. J Neurosci 14:3805-3815.

Rodriguez AR, de Sevilla Müller LP, Brecha NC (2014) The RNA binding protein RBPMS is a selective marker of ganglion cells in the mammalian retina. J Comp Neurol 522:1411-1443.

Román Rosón M, Bauer Y, Kotkat AH, Berens P, Euler T, Busse L (2019) Mouse dLGN receives functional input from a diverse population of retinal ganglion cells with limited convergence. Neuron 102:462-476.e8.

Rompani SB, Müllner FE, Wanner A, Zhang C, Roth CN, Yonehara K, Roska B (2017) Different modes of visual integration in the lateral geniculate nucleus revealed by single-cell-initiated transsynaptic tracing. Neuron 93:767-776.e6.

Roy K, Kumar S, Bloomfield SA (2017) Gap junctional coupling between retinal amacrine and ganglion cells underlies coherent activity integral to global object perception. Proc Natl Acad Sci U S A 114:E10484-E10493.

Schindelin J, Arganda-Carreras I, Frise E, Kaynig V, Longair M, Pietzsch T, Preibisch S, Rueden C, Saalfeld S, Schmid B, Tinevez JY, White DJ, Hartenstein V, Eliceiri K, Tomancak P, Cardona A (2012) Fiji: an opensource platform for biological-image analysis. Nat Methods 9:676-682.

Schmidt TM, Alam NM, Chen S, Kofuji P, Li W, Prusky GT, Hattar S (2014) A role for melanopsin in alpha retinal ganglion cells and contrast detection. Neuron 82:781-788.

Schubert T, Maxeiner S, Krüger O, Willecke K, Weiler R (2005a) Connexin 45 mediates gap junctional coupling of bistratified ganglion cells in the mouse retina. J Comp Neurol 490:29-39.

Schubert T, Degen J, Willecke K, Hormuzdi SG, Monyer H, Weiler R (2005b) Connexin36 mediates gap junctional coupling of alphaganglion cells in mouse retina. J Comp Neurol 485:191-201.

Traub RD, Whittington MA, Gutiérrez R, Draguhn A (2018) Electrical coupling between hippocampal neurons: contrasting roles of principal cell gap junctions and interneuron gap junctions. Cell Tissue Res 373:671-691.

Trenholm S, Schwab DJ, Balasubramanian V, Awatramani GB (2013) Lag normalization in an electrically coupled neural network. Nat Neurosci 16:154-156.

Trenholm S, McLaughlin AJ, Schwab DJ, Turner MH, Smith RG, Rieke F, Awatramani GB (2014) Nonlinear dendritic integration of electrical and chemical synaptic inputs drives fine-scale correlations. Nat Neurosci 17:1759-1766.

Trong PK, Rieke F (2008) Origin of correlated activity between parasol retinal ganglion cells. Nat Neurosci 11:1343-1351.

Vaney DI (1991) Many diverse types of retinal neurons show tracer coupling when injected with biocytin or neurobiotin. Neurosci Lett 125:187-190.

Veruki ML, Hartveit E (2002) AII (Rod) amacrine cells form a network of electrically coupled interneurons in the mammalian retina. Neuron 33:935-946.

Völgyi B, Abrams J, Paul DL, Bloomfield SA (2005) Morphology and tracer coupling pattern of alpha ganglion cells in the mouse retina. J Comp Neurol 492:66-77.

Völgyi B, Pan F, Paul DL, Wang JT, Huberman AD, Bloomfield SA (2013) Gap junctions are essential for generating the correlated spike activity of neighboring retinal ganglion cells. PLoS One 8:e69426.

Wässle H (2004) Parallel processing in the mammalian retina. Nat Rev Neurosci 5:747-757.

Wässle H, Peichl L, Boycott BB (1981) Dendritic territories of cat retinal ganglion cells. Nature 292:344-345.

Xin D, Bloomfield SA (1997) Tracer coupling pattern of amacrine and ganglion cells in the rabbit retina. J Comp Neurol 383:512-528.

Yao X, Cafaro J, McLaughlin AJ, Postma FR, Paul DL, Awatramani G, Field GD (2018) Gap junctions contribute to differential light adaptation across direction-selective retinal ganglion cells. Neuron 100:216-228.e6.

Zaghloul KA, Boahen K, Demb JB (2003) Different circuits for ON and OFF retinal ganglion cells cause different contrast sensitivities. J Neurosci 23:2645-2654.

Zhang J, Li W, Hoshi H, Mills SL, Massey SC (2005) Stratification of alpha ganglion cells and ON/OFF directionally selective ganglion cells in the rabbit retina. Vis Neurosci 22:535-549. 